Bundesgesundheitsbl - Gesundheitsforsch Gesundheitsschutz 2007 ·50:711-717 DOI 10.1007/s00103-007-0232-8

c) Springer Medizin Verlag 2007

\author{
U. Ellert $\cdot$ H. Neuhauser $\cdot$ A. Roth-Isigkeit \\ Robert Koch-Institut, Berlin, BRD
}

\title{
Schmerzen bei Kindern und Jugendlichen in Deutschland: Prävalenz und Inanspruch- nahme medizinischer Leistungen
}

\section{Ergebnisse des Kinder- und Jugendgesund- heitssurveys (KiGGS)}

\section{Hintergrund}

Schmerzen sind auch im Kindes- und Jugendalter eine häufige Erfahrung [1, $2,3,4]$. Für Deutschland fehlen bislang repräsentative epidemiologische Untersuchungen, die verlässliche Schätzungen der Prävalenz von Schmerzen bei Kindern und Jugendlichen erlauben. Insbesondere vor dem Hintergrund, dass die $12-\mathrm{Mo}-$ nats-Prävalenz von Schmerzen bei Erwachsenen in Deutschland mit $91 \%$ sehr hoch ist [5] und mit dem Alter zunimmt, stellt sich die Frage, ob Schmerzen auch schon bei Kindern ein so allgegenwärtiges Problem darstellen.

In bisherigen epidemiologischen Studien über Schmerzen bei Kindern und Jugendlichen wurden vornehmlich spezifische Schmerzsyndrome (z. B. Kopfschmerzen, Bauchschmerzen, Rückenschmerzen) analysiert $[6,7,8]$. Daher ist es Ziel der vorliegenden Untersuchung, die Prävalenz von Schmerzen im Allgemeinen und umfassend differenziert nach Schmerzlokalisationen bei deutschen Kindern und Jugendlichen im Altersspektrum von 3-17 Jahren anzugeben.

Vergleichsweise hohe Prävalenzraten für Schmerzsyndrome im Kindesalter wurden in internationalen epidemiologischen Untersuchungen berichtet. Nach den Ergebnissen einer repräsentativen niederländischen Studie berichteten $54 \%$ der befragten Kinder und Jugendlichen im Alter von o-18 Jahren ( $\mathrm{N}=5424)$, in den letzten 3 Monaten Schmerzen erfahren zu haben. $50 \%$ dieser Kinder und Jugendlichen gaben mehrere Schmerzlokalisationen an, am häufigsten Kombinationen von Rücken-, Kopf- und Bauchschmerzen. 25\% der Kinder und Jugendlichen dieser Untersuchung berichteten Schmerzen über einen Zeitraum von länger als 3 Monaten. $57 \%$ dieser Kinder konsultierten im $\mathrm{Zu}$ sammenhang mit dem Schmerzproblem einen Arzt, 39 \% setzten Medikamente zur Schmerzbehandlung ein [9].

Die nach dem gegenwärtigen Stand der Forschung im Bereich Schmerzen bei Kindern und Jugendlichen bestehenden Datenlücken in Deutschland sollten durch den Studienteil „Schmerzen“ des Kinderund Jugendgesundheitssurveys (KiGGS) geschlossen werden. Dabei wurden Merkmale von Schmerzen im Kindes- und Jugendalter (z. B. Lokalisation, Häufigkeit, Intensität) allgemein, multiple Schmerzlokalisationen und die Inanspruchnahme von Leistungen des Gesundheitswesens
(Arztbesuche und Medikamenteneinnahmen wegen Schmerzen) erhoben.

Da Schmerz nicht nur Konsequenzen für die Lebensqualität des einzelnen akut oder chronisch von Schmerz Betroffenen hat, sondern darüber hinaus Anforderungen an das Gesundheitssystem stellt, sollen mit dieser aktuellen und repräsentativen Datenbasis folgende Fragestellungen beantwortet werden:

1. Wie häufig sind Schmerzen bei Kindern und Jugendlichen?

2. Gibt es alters- und geschlechtsdifferente Schmerzlokalisationen bei Kindern und Jugendlichen?

3. In welchem Ausmaß führen Schmerzen bei Kindern und Jugendlichen zu einer Inanspruchnahme des Gesundheitssystems (Arztbesuche, Einnahme von Medikamenten)?

\section{Methoden}

Konzept, Design und Durchführung des KiGGS werden an anderer Stelle in diesem Heft ausführlich beschrieben [10, 11, 12]. Die KiGGS-Studie wurde von Mai 2003 bis Mai 2006 vom Robert KochInstitut (RKI) durchgeführt. Ziel dieses bundesweiten Befragungs- und Unter- 
suchungssurveys war es, erstmals umfassende und bundesweit repräsentative Daten zum Gesundheitszustand von Kindern und Jugendlichen im Alter von o17 Jahren zu erheben. An der Studie haben insgesamt 17.641 Kinder und Jugendliche (8656 Mädchen und 8985 Jungen) aus 167 für die Bundesrepublik repräsentativen Städten und Gemeinden teilgenommen. Die Teilnahmequote betrug $66,6 \%$.

Durch den Fragebogenteil „Schmerzen“ des KiGGS wurden die 3-MonatsSchmerzprävalenz, Lokalisation, Auftretenshäufigkeit, Intensität und der Zeitraum seit dem ersten Auftreten von Schmerzen erfasst. Darüber hinaus wurden selbst wahrgenommene Einflussfaktoren auf die Entstehung von Schmerzen sowie Folgen und Beeinträchtigungen durch Schmerzen im alltäglichen Leben und die Inanspruchnahme von $\mathrm{Ge}$ sundheitsdienstleistungen (Arztbesuche und Medikamenteneinnahmen) wegen Schmerzen erhoben. Für 3- bis 10-jährige Kinder beantworteten die Eltern die Fragen zu Schmerzen (Fremdeinschätzung). 11- bis 17-jährige Kinder und Jugendliche beantworteten die Fragen zu Schmerzen selbst (Selbsteinschätzung). Aus diesem Grunde werden die Ergebnisse für diese beiden Gruppen getrennt berichtet.

Wenn die Antwort auf die erste schmerzbezogene Frage: „Hattest Du/ hatte Ihr Kind in den letzten 3 Monaten Schmerzen?“ - „nein“ lautete, waren keine weiteren schmerzbezogenen Fragen zu beantworten. Wenn die Antwort ,ja“ lautete, wurde zunächst die Lokalisation der Schmerzen in diesem Zeitraum erfragt. Die Kinder und Jugendlichen bzw. deren Eltern wurden gebeten, aus einer Liste möglicher Schmerzlokalisationen (Kopf, Rücken, Ohren, Augen, Bauch, Unterleib, Arm, Bein, Brustkorb, Hals, Zahn oder andere/welche; bei Mädchen wurde zusätzlich der Regelschmerz erfasst) anzugeben, ob sie an der jeweiligen Lokalisation in den letzten 3 Monaten Schmerzen gehabt haben, wenn ,ja“, ob dies einmalig oder wiederholt der Fall gewesen sei.

Im Anschluss daran wurde erfragt, welcher dieser Schmerzen der Hauptschmerz gewesen sei. Für den Schmerz, der als Hauptschmerz eingestuft wurde, wurde gebeten, die Häufigkeit in den letzten 3 Monaten (ein einziges Mal, unre-

Tabelle 1

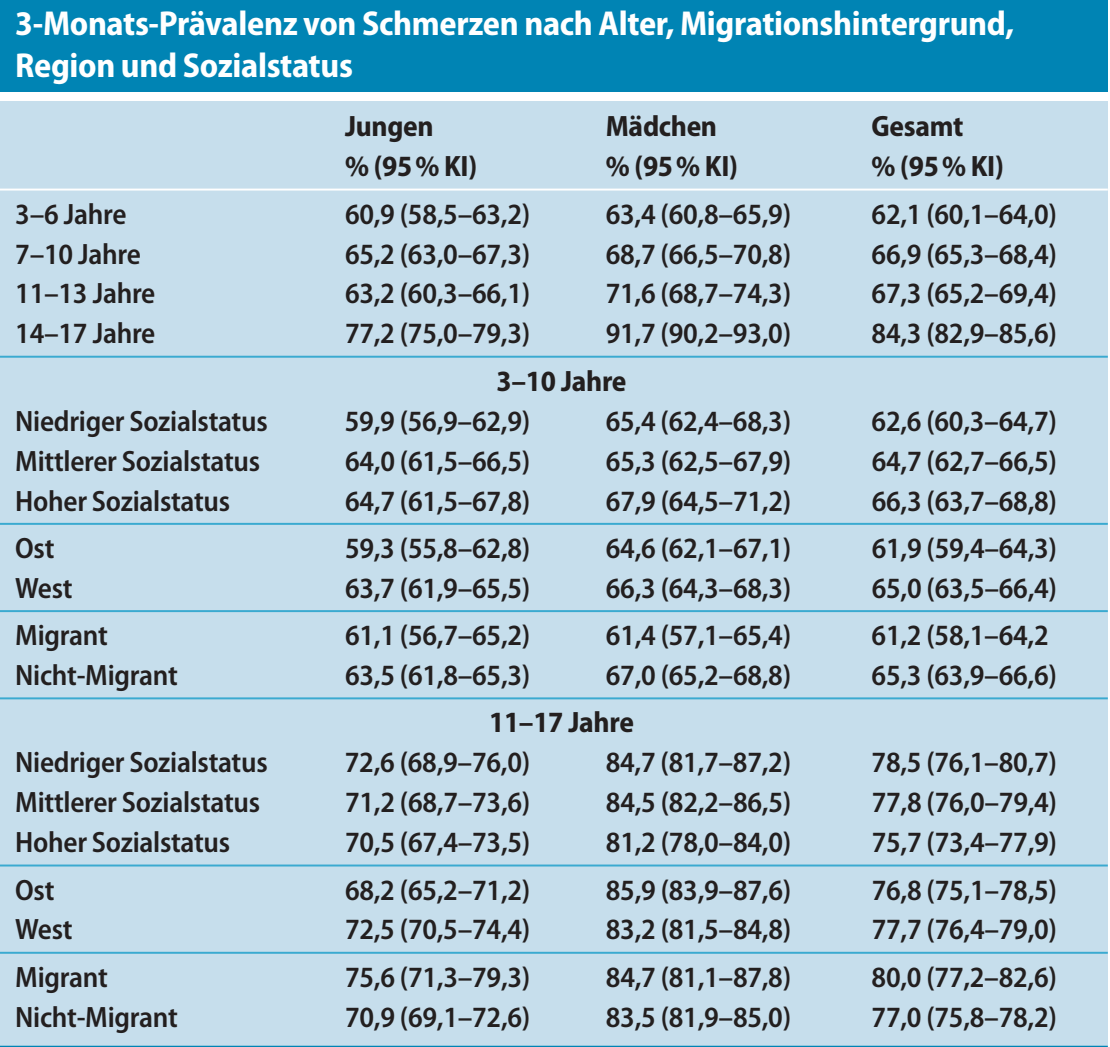

gelmäßig insgesamt xx-mal in den letzten 3 Monaten oder regelmäßig wiederkehrend: einmal pro Monat, 2- bis 3-mal pro Monat, einmal in der Woche, mehrmals in der Woche, täglich), die Intensität und den Zeitraum des erstmaligen Auftretens (im letzten Monat, im letzten Vierteljahr, im letzten halben Jahr, in den letzten 12 Monaten, vor mehr als einem Jahr) anzugeben. Die Schmerzintensität wurde mit visueller Analogskala mit den verbalen Endpunkten „kaum spürbare Schmerzen“vs. „stärkste vorstellbare Schmerzen“ mit der Frage: „Wie stark ist der Hauptschmerz (normalerweise)?" erhoben.

Mittels einer vorgegebenen Liste und freier Beantwortungsmöglichkeit wurden Faktoren erfragt, mit denen das Auftreten des Hauptschmerzes nach Wahrnehmung der Befragten im Zusammenhang stand. Art und Ausmaß von Beeinträchtigungen durch Schmerzen in den Bereichen Schlafen, Essen, Schulabwesenheit, Hobbys, Sozialkontakte und Inanspruchnahme des Gesundheitswesens (Arztbesuche und Medikamenteneinnahmen) wurden mit vorgegebenen Antwortkategorien erfragt (nie, einmal, manchmal, häufig, immer).

\section{Statistische Analysen}

Um repräsentative Aussagen treffen zu können, wurden die Analysen mit einem Gewichtungsfaktor durchgeführt, der Abweichungen der Netto-Stichprobe von der Bevölkerungsstruktur (Stand: 31.12.2004) hinsichtlich Alter (in Jahren), Geschlecht, Region (Ost/West/Berlin) und Staatsangehörigkeit korrigiert. Um die Korrelation der Probanden innerhalb einer Gemeinde zu berücksichtigen, wurden die Konfidenzintervalle und die p-Werte mit den SPSS14-Verfahren für komplexe Stichproben bestimmt. Gruppenunterschiede mit p-Werten von kleiner als 0,05 oder mit $95 \%$-Konfidenzintervallen, die sich nicht überschneiden, werden als statistisch signifikant gewertet.

\section{Ergebnisse}

In die Auswertungen einbezogen wurden Angaben von Kindern und Jugendlichen, für die die Beantwortung der Filterfrage („Hattest Du/Hatte Ihr Kind in den letzten 3 Monaten Schmerzen?") vorlagen. Das waren $98 \%(\mathrm{~N}=14.959)$ der 3- bis 17 -jäh- 
rigen Studienteilnehmer. In den soziodemographischen Merkmalen unterscheidet sich die Untersuchungsstichprobe nicht von der gesamten Studienpopulation, die in Lange $\mathrm{M}$ et al. [13] ausführlich beschrieben ist.

\section{3-Monats-Schmerzprävalenz}

10.640 von 14.959 Kindern und Jugendlichen $(71 \%)$ hatten in den letzten $3 \mathrm{Mo}$ naten Schmerzen. In der Gruppe der 3 - bis 10-jährigen Kinder waren es 64,5 \% (66,1 \% der Mädchen; 63,1 \% der Jungen). Mehr als drei Viertel (77,6\%; Mädchen 83,7\%, Jungen $71,7 \%$ ) der 11 - bis 17 -jährigen Kinder und Jugendlichen bejahten das Auftreten von Schmerzen in den letzten 3 Monaten. Die 3-Monats-Prävalenz von Schmerzen nahm signifikant mit dem Alter zu. Mädchen berichteten in allen Altersgruppen signifikant mehr Schmerzen als Jungen (- Tabelle 1).

Signifikante Unterschiede nach sozialer Herkunft bezüglich der Prävalenz von Schmerzen waren bei den älteren Studienteilnehmern nicht zu beobachten. Bei den 3- bis 10-Jährigen wurden mit höherem sozialen Status häufiger Schmerzen berichtet. Dies galt für Jungen und Mädchen, war allerdings nur für die Jungen signifikant.

Für Kinder der Altersgruppe 3-10 Jahre aus den alten Bundesländern wurden häufiger Schmerzen als für gleichaltrige Kinder aus den neuen Bundesländern (inklusive Berlin) berichtet ( $\bullet$ Tabelle 1). Signifikant war dieser Unterschied in der Gesamtgruppe und bei den Jungen. Bei den Älteren (11- bis 17-Jährige) ergaben sich in der Gesamtgruppe keine signifikanten Ost-West-Unterschiede.

Differenziert nach Migrationshintergrund der Kinder und Jugendlichen ergab sich zwischen den beiden Altersgruppen ein ungleiches Bild. Die Eltern von 3- bis 10-jährigen Kindern ohne Migrationshintergrund gaben signifikant häufiger Schmerzen bei ihren Kindern an als Eltern gleichaltriger Kinder mit Migrationshintergrund (• Tabelle 1). Bei den Jungen allein war dieser Unterschied allerdings nicht signifikant. Bei den 11- bis 17-Jährigen gaben Kinder und Jugendliche mit Migrationshintergrund signifikant häufiger Schmerzen an als Kinder und

Bundesgesundheitsbl - Gesundheitsforsch - Gesundheitsschutz 2007 · 50:711-717

DOI 10.1007/s00103-007-0232-8

(c) Springer Medizin Verlag 2007

U. Ellert $\cdot$ H. Neuhauser $\cdot$ A. Roth-Isigkeit

Schmerzen bei Kindern und Jugendlichen in Deutschland: Prävalenz und Inanspruchnahme medizinischer Leistungen. Ergebnisse des Kinder- und Jugendgesundheitssurveys (KiGGS)

\section{Zusammenfassung}

Im Rahmen des Kinder- und Jugendgesundheitssurveys (KiGGS) wurden repräsentative Daten zu Schmerzen bei 14.959 Kindern und Jugendlichen im Alter von 317 Jahren in Deutschland erhoben. Die Ergebnisse werden getrennt berichtet für 11bis 17-Jährige, die selbst befragt wurden, und für die 3- bis 10-Jährigen, über deren Schmerzen die Eltern berichteten. Die 3-Monats-Prävalenz von Schmerzen betrug in der Gruppe der 3- bis 10-Jährigen $64,5 \%$ und in der Gruppe der 11- bis 17Jährigen 77,6\% (insgesamt für die 3- bis 17-jährigen Kinder 71,1\%). Die Schmerzprävalenz nahm signifikant mit dem Alter zu, Mädchen berichteten in allen Altersgruppen signifikant häufiger über Schmerzen als gleichaltrige Jungen. Häufigste Schmerzlokalisationen war bei den 3- bis 10-Jährigen der Bauch gefolgt von Kopf und Hals. Kinder und Jugendliche im Alter von 11-17 Jahren berichteten am häu-

figsten über Kopfschmerzen, gefolgt von Bauch- und Rückenschmerzen. Schmerzen, die mindestens einmal in der Woche in den letzten 3 Monaten auftraten, wurden für 24,3\% der 11- bis 17-jährigen Kinder und Jugendlichen und für 9,9\% der 3- bis 10-Jährigen Kinder mit Schmerzen angegeben. Mehr als die Hälfte $(54,1 \%)$ der 3- bis 10-Jährigen und mehr als ein Drittel der 11- bis 17-Jährigen (35,9\%) mit Angaben zu wiederkehrenden Hauptschmerzen konsultierten deswegen einen Arzt, Medikamente nahmen jeweils 36,7\% bzw. 46,7\% der Schmerzbetroffenen. Die Ergebnisse belegen, dass Schmerzen bei Kindern und Jugendlichen ein ernst zu nehmendes Problem darstellen.

\section{Schlüsselwörter}

Gesundheitssurvey · Kinder · Jugendliche - Schmerzen - Epidemiologie · Inanspruchnahme medizinischer Leistungen

\section{Pain in children and adolescents in Germany: the prevalence and usage of medical services. Results of the German Health Interview and Examination Survey for Children and Adolescents (KiGGS)}

\section{Abstract}

As part of the German Health Interview and Examination Survey for Children and Adolescents (KiGGS), representative data were collected on pain in 14,959 children and adolescents aged 3 to 17 years in Germany. The results are reported separately for 1117-year olds, who were asked themselves, and 3-10 year olds, whose parents reported on their pain. Among the 3-10 year olds, the prevalence of pain over three months was $64.5 \%$ and among the $11-17$ year olds it was $77.6 \%$ (71.1\% altogether for the 3-17 year old children). Pain prevalence increased significantly with age; in all age groups girls reported pain significantly more often than boys of the same age. In the 3-10 year olds the most common pain localisation was stomach pains, followed by pain in the head and throat. Children and adolescents aged 11 to 17 most often reported headaches, followed by pains in the stomach and back. Pain occurring at least once a week in the last three months was reported by $24.3 \%$ of the 11-17 year old children and adolescents and by $9.9 \%$ of the $3-10$ year old children suffering from pain. More than half (54.1\%) of the 3-10 year olds and more than one third of the 11-17 year olds (35.9\%) who reported recurrent principal pain consulted a doctor for this reason; $36.7 \%$ and $46.7 \%$ respectively took medicine. These results show that pain is a relevant problem in children and adolescents in Germany.

\section{Keywords}

Health Survey · Children · Adolescents · Pain · Epidemiology · Use of medical services 
Jugendliche gleichen Alters ohne Migrationshintergrund, dieser Unterschied war bei den Mädchen nicht signifikant.

\section{Schmerzlokalisationen}

Nach Angaben der Eltern klagten 3- bis 10-jährige Kinder am häufigsten über Bauchschmerzen (69\%), gefolgt von Kopf- (56\%), Hals- (48\%), Bein- $(36 \%)$ und Ohrenschmerzen (29\%). Von den 11bis 17-jährigen befragten Kindern und Jugendlichen wurden die Schmerzlokalisationen Kopf (78\%), Bauch (6o\%), Rücken (49\%), Hals (45\%) und Bein (36\%) in dieser Reihenfolge am häufigsten genannt.

Für die in $\bullet$ Abb. $1 \mathrm{a}$ und $\mathbf{1 b}$ gezeigte Darstellung der Altersabhängigkeit der verschiedenen Schmerzen wurden einmalige Schmerzen in den jeweiligen Lokalisationen ausgeschlossen, um die Heterogenität des Schweregrades der angegebenen Schmerzen etwas zu reduzieren. Wiederholte Kopf-, Regel-, Unterleibs- und Rückenschmerzen nahmen bei Mädchen signifikant mit dem Alter zu. Demgegenüber nahmen bei den Jungen lediglich Kopf- und Rückenschmerzen signifikant im Altersgang zu.

Schmerzen an mehr als einer Lokalisation in den letzten 3 Monaten hatten mehr als die Hälfte $(54,7 \%)$ aller befragten Kinder. Lag die Prävalenz für Schmerzen an mehreren Körperregionen bei den 3 bis 10-Jährigen noch bei $44,6 \%$, so waren es in der Gruppe der 11- bis 17-Jährigen schon fast zwei Drittel $(64,5 \%)$, die mehrere Schmerzlokalisationen angaben.

\section{Hauptschmerz}

In der Gruppe der 3- bis 10-jährigen Kinder wurden als Hauptschmerz am häufigsten Bauchschmerzen (33\%) gefolgt von Kopf- (20\%), Bein- (14\%), Hals(11\%) und Ohrenschmerzen (10\%) angegeben. Signifikante geschlechtsspezifische Unterschiede in den Hauptschmerzlokalisationen bestanden in dieser Altersgruppe nicht.

Mehr als ein Drittel (34\%) der 11- bis 17-jährigen Kinder und Jugendlichen gibt an, dass Kopfschmerzen in den letzten 3 Monaten ihr Hauptschmerz gewesen sei. Daran schlossen sich mit großem Abstand Rücken- (13\%), Bein- (12\%) und
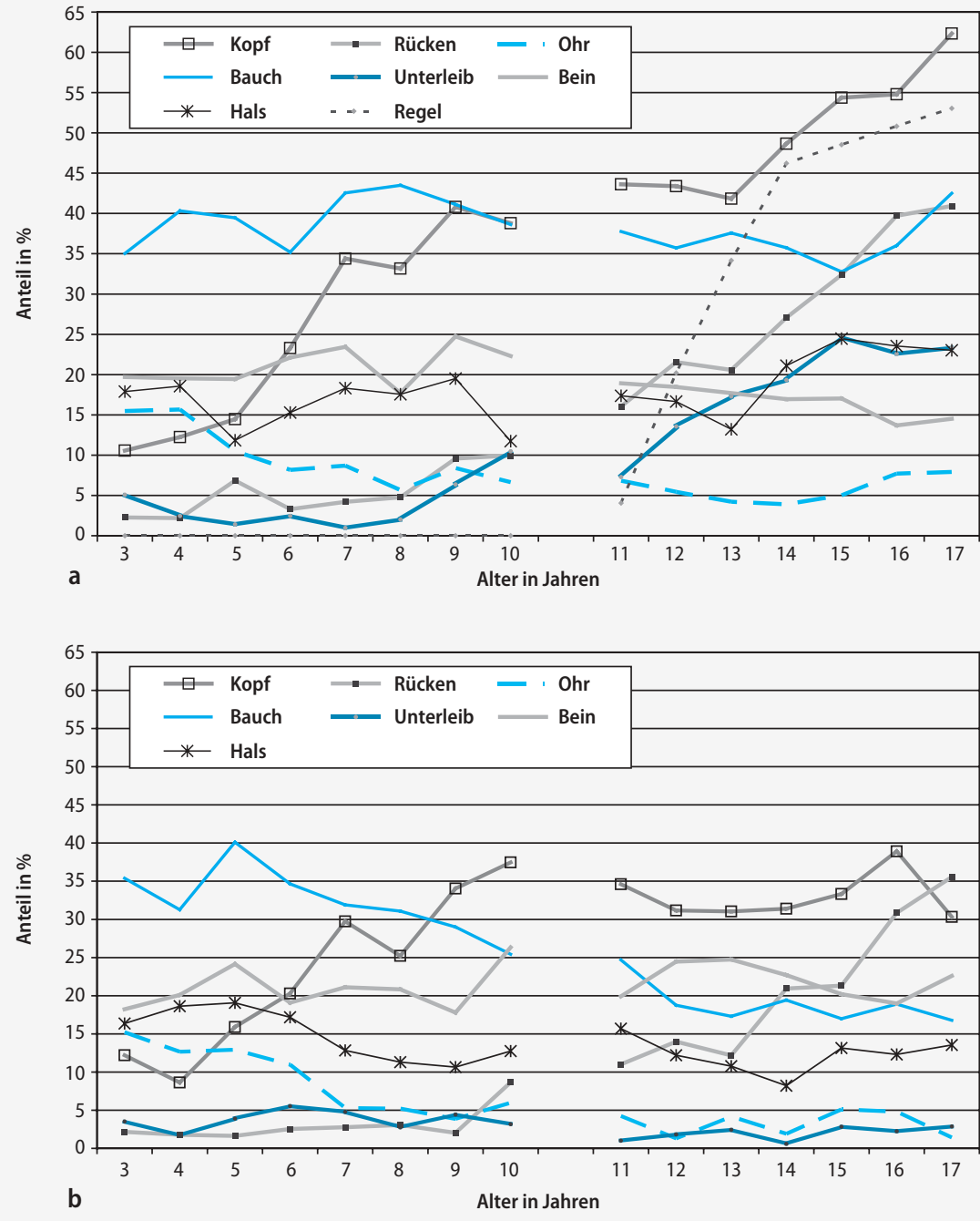

Abb. $1 \Delta$ a Schmerzlokalisationen in den letzten 3 Monaten (wiederholter Schmerz) nach Alter bei Mädchen. $b$ Schmerzlokalisationen in den letzten 3 Monaten (wiederholter Schmerz) nach Alter bei Jungen

Bauchschmerzen (12\%) als Hauptschmerz in den letzten 3 Monaten an. Während der Kopf sowohl von den Jungen als auch den Mädchen am häufigsten als Hauptschmerzlokalisation genannt wird, ist die weitere Rangfolge der Lokalisationen bei Jungen an zweiter Stelle Bein (18\%) gefolgt von Rücken (15\%) und Bauch (12\%). $17 \%$ der befragten 11 - bis 17 -jährigen Mädchen bezeichneten Regelschmerzen als ihren Hauptschmerz, damit war der Regelschmerz die zweithäufigste Hauptschmerznennung bei den Mädchen, gefolgt von Bauch- (13\%) und Rückenschmerzen (11\%).

\section{Wiederkehrende Schmerzen}

Bei 30,6\% der 3- bis 10-jährigen Kinder berichteten deren Eltern wiederkehrende Schmerzen in den letzten 3 Monaten. Einmal pro Woche oder häufiger traten die Schmerzen bei 9,9\% der 3- bis 10-jährigen Kinder auf. Mehr als die Hälfte (52,9\%) der befragten 11- bis 17-jährigen Kinder und Jugendlichen berichteten wiederkehrende Schmerzen. 24,3\% der Kinder und Jugendlichen im Alter von 11-17 Jahren berichteten, dass ihre Schmerzen einmal pro Woche oder häufiger aufgetreten seien. Mit zunehmendem Alter der Kinder und Jugendlichen traten die Schmerzen deutlich häufiger wiederkehrend auf. Mädchen berichteten in allen Altersstufen signifikant häufiger wiederkehren- 
Tabelle 2

Auftretenshäufigkeit von Schmerzen in den letzten 3 Monaten

\begin{tabular}{|c|c|c|c|c|c|c|}
\hline & \multicolumn{6}{|l|}{ Häufigkeit } \\
\hline & $\begin{array}{l}\text { Ein einziges Mal } \\
\%(\mathbf{K I})\end{array}$ & $\begin{array}{l}\text { Einmal im Monat } \\
\% \text { (KI) }\end{array}$ & $\begin{array}{l}\text { 2-bis 3-mal im Monat } \\
\% \text { (KI) }\end{array}$ & $\begin{array}{l}\text { Einmal pro Woche } \\
\%(\mathrm{KI})\end{array}$ & $\begin{array}{l}\text { Mehrmals pro Woche } \\
\%(\mathrm{KI})\end{array}$ & $\begin{array}{l}\text { Täglich } \\
\% \text { (KI) }\end{array}$ \\
\hline \multicolumn{7}{|l|}{ 3-6 } \\
\hline Jungen & $21,6(19,6-23,6)$ & $7,3(6,0-8,9)$ & $11,0(9,5-12,8)$ & $2,0(1,4-2,9)$ & $5,5(4,6-6,7)$ & $0,9(0,5-1,5)$ \\
\hline Mädchen & $20,3(18,2-22,5)$ & $8,1(7,0-9,4)$ & $10,7(9,2-12,4)$ & $3,6(2,9-4,6)$ & $5,2(4,2-6,6)$ & $0,9(0,5-1,5)$ \\
\hline \multicolumn{7}{|l|}{$7-10$} \\
\hline Jungen & $20,8(19,0-22,7)$ & $8,7(7,5-10,0)$ & $12,2(10,7-13,8)$ & $3,6(2,8-4,6)$ & $5,1(4,1-6,4)$ & $0,7(0,4-1,2)$ \\
\hline Mädchen & $17,6(15,9-19,5)$ & $8,0(6,7-9,4)$ & $17,1(15,3-19,0)$ & $5,6(4,5-6,8)$ & $6,0(4,9-7,3)$ & $0,6(0,4-1,1)$ \\
\hline $3-10$ & $20,1(19,2-21,0)$ & $8,0(7,4-8,7)$ & $12,7(12,0-13,6)$ & $3,7(3,3-4,1)$ & $5,5(4,9-6,1)$ & $0,7(0,6-1,0)$ \\
\hline \multicolumn{7}{|l|}{$11-13$} \\
\hline Jungen & $19,3(17,3-21,5)$ & $8,4(6,8-10,2)$ & $11,8(10,1-13,7)$ & $6,3(5,1-7,8)$ & $9,2(7,6-11,0)$ & $2,4(1,7-3,4)$ \\
\hline Mädchen & $13,5(11,7-15,4)$ & $9,9(8,3-11,8)$ & $14,9(12,8-17,2)$ & $9,1(7,5-11,0)$ & $14,4(12,5-16,6)$ & $4,2(3,2-5,3)$ \\
\hline \multicolumn{7}{|l|}{$14-17$} \\
\hline Jungen & $25,0(22,9-27,2)$ & $9,6(8,4-11,1)$ & $14,5(12,7-16,4)$ & $6,8(5,7-8,0)$ & $9,4(8,1-10,9)$ & $3,3(2,5-4,4)$ \\
\hline Mädchen & $12,3(10,7-14,0)$ & $21,6(19,6-23,8)$ & $20,0(18,0-22,1)$ & $9,6(8,1-11,3)$ & $17,2(15,5-19,1)$ & $4,8(3,8-6,1)$ \\
\hline $11-17$ & $17,9(16,9-18,9)$ & $13,0(12,1-13,9)$ & $15,6(14,6-16,7)$ & $8,0(7,3-8,7)$ & $12,6(11,8-13,5)$ & $3,7(3,2-4,3)$ \\
\hline
\end{tabular}

Tabelle 3

Erstmaliges Auftreten des Hauptschmerzes

\begin{tabular}{|c|c|c|c|c|c|}
\hline & \multicolumn{5}{|l|}{ Erstes Auftreten } \\
\hline & $\begin{array}{l}\text { Im letzten } \\
\text { Monat } \\
\% \text { (KI) }\end{array}$ & $\begin{array}{l}\text { In den letzten } \\
3 \text { Monaten } \\
\% \text { (KI) }\end{array}$ & $\begin{array}{l}\text { In den letzten } \\
6 \text { Monaten } \\
\%(\mathrm{KI})\end{array}$ & $\begin{array}{l}\text { In den letzten } \\
12 \text { Monaten } \\
\% \text { (KI) }\end{array}$ & $\begin{array}{l}\text { Vor mehr als } \\
\text { einem Jahr } \\
\% \text { (KI) }\end{array}$ \\
\hline \multicolumn{6}{|l|}{$3-6$} \\
\hline Jungen & $14,5(11,6-17,9)$ & $18,0(14,8-21,7)$ & $14,4(11,5-17,8)$ & $14,5(11,6-18,1)$ & $38,6(34,0-43,4)$ \\
\hline Mädchen & $13,3(10,2-17,0)$ & $18,0(15,2-21,2)$ & $12,7(10,2-15,8)$ & $14,6(11,9-17,9)$ & $41,3(36,9-46,0)$ \\
\hline \multicolumn{6}{|l|}{$7-10$} \\
\hline Jungen & $10,9(8,8-13,5)$ & $17,7(14,8-21,0)$ & $12,3(10,1-15,1)$ & $13,3(11,0-16,0)$ & $45,8(42,0-49,8)$ \\
\hline Mädchen & $10,0(7,9-12,6)$ & $16,4(13,6-19,6)$ & $15,1(13,0-17,5)$ & $13,4(11,0-16,2)$ & $45,1(41,3-48,9)$ \\
\hline $3-10$ & $12,0(10,6-13,5)$ & $17,4(15,9-19,1)$ & $13,7(12,4-15,1)$ & $13,9(12,5-15,4)$ & $43,0(40,7-45,4)$ \\
\hline \multicolumn{6}{|l|}{$11-13$} \\
\hline Jungen & $39,9(35,6-44,5)$ & $17,2(14,2-20,7)$ & $9,7(7,3-12,8)$ & $4,1(2,7-6,2)$ & $29,0(25,5-32,7)$ \\
\hline Mädchen & $34,3(30,8-38,0)$ & $17,4(14,6-20,6)$ & $10,1(8,0-12,7)$ & $6,2(4,6-8,4)$ & $32,0(28,7-35,4)$ \\
\hline \multicolumn{6}{|l|}{$14-17$} \\
\hline Jungen & $16,4(13,8-19,4)$ & $16,9(14,7-19,3)$ & $12,8(10,7-15,3)$ & $6,8(5,2-8,7)$ & $47,1(43,7-50,5)$ \\
\hline Mädchen & $14,2(12,4-16,3)$ & $11,8(10,0-13,9)$ & $9,6(8,1-11,4)$ & $8,2(6,7-10,0)$ & $56,1(53,3-58,9)$ \\
\hline $11-17$ & $22,3(21,0-23,6)$ & $15,0(13,8-16,2)$ & $10,6(9,5-11,8)$ & $6,9(6,0-7,9)$ & $45,3(43,6-47,0)$ \\
\hline
\end{tabular}

de Schmerzen als gleichaltrige Jungen (- Tabelle 2).

\section{Erstmaliges Auftreten des Hauptschmerzes}

Bei $43 \%$ der 3- bis 10-jährigen Kinder traten die Hauptschmerzen schon vor mehr als einem Jahr erstmalig auf. Nur $12 \%$ der Eltern berichteten, dass die Schmerzen ihrer Kinder im Alter von 3-10 Jahren erst seit einem Monat aufträten. Insgesamt gaben auch in der Gruppe der 11bis 17-jährigen Kinder und Jugendlichen ähnlich viele $(45 \%)$ an, das erstmalige Auftreten ihrer Schmerzen liege mehr als ein Jahr zurück. Allerdings ist innerhalb dieser Altersgruppe ein starker Altersgang zu verzeichnen, je älter die Kinder und Jugendlichen werden, desto häufiger berichten sie, das erstmalige Auftreten ihrer Schmerzen läge schon länger als ein Jahr zurück. Im Gegensatz dazu nahm der Anteil derer, die ihren Schmerz erst vor einem Monat erstmalig feststellten, bei den 13- bis 17-Jährigen mit höherem Alter ab (• Tabelle 3). 
Inanspruchnahme des Gesundheitssystems wegen wiederkehrender Hauptschmerzen

Mehr als die Hälfte $(54,1 \%)$ aller Eltern von 3- bis 10-jährigen Kindern mit Angaben zu wiederkehrenden Hauptschmerzen berichteten, deswegen einen Arzt konsultiert zu haben; 14,9\% sagten, dass das manchmal und 13,9\% häufig oder immer der Fall gewesen sei. 36,7\% der Eltern gaben an, dass ihre Kinder wegen Schmerzen Medikamente eingenommen hätten, von 18,5\% der Kinder wurden Medikamente „manchmal“ eingenommen, „häufig“ oder „immer“ von 9,6\%.

Bei den 11- bis 17-jährigen Kindern und Jugendlichen mit Schmerzen waren es mehr als ein Drittel (35,9\%), die berichteten, deswegen einen Arzt konsultiert zu haben, jeder Zehnte konsultierte manchmal und 8,2\% häufig oder immer einen Arzt. 46,7\% der 11- bis 17-Jährigen gaben an, wegen der Schmerzen Medikamente eingenommen zu haben, knapp ein Viertel $(24,4 \%)$ tat dies manchmal, $11,4 \%$ häufig oder immer.

\section{Diskussion}

Mehr als zwei Drittel der 3- bis 10-Jährigen und sogar mehr als drei Viertel der 11- bis 17-Jährigen hatten in den letzten 3 Monaten Schmerzen. Diese Ergebnisse befinden sich in weitgehender Übereinstimmung mit den Studien anderer Arbeitsgruppen $[1,2]$. Nun könnte man auf den Gedanken kommen, dass sich Kinder beim Spielen häufig stoßen oder wehtun, ohne sich wirklich schlimm zu verletzen und deshalb Schmerz auch eine alltägliche, aber harmlose Erfahrung ist. Das mag für einen Teil der Schmerzangaben durchaus zutreffen, ein überraschend hoher Anteil der berichteten Schmerzen führte aber zu wiederholter Inanspruchnahme ärztlicher Leistungen und/oder häufigem Medikamentenkonsum. Bei etwa einem Drittel der 3- bis 10-jährigen Kinder und mehr als der Hälfte der 11- bis 17-jährigen Kinder und Jugendlichen kehrten die Schmerzen wieder.

Unterschiede nach sozialer Herkunft, Migrationshintergrund und dem Leben in den alten oder neuen Bundesländern bezüglich der Prävalenz von Schmerzen deuten sich nach dieser ersten Analyse an, sollten jedoch in vertiefenden Untersuchungen genauer überprüft werden.

\section{Altersabhängigkeit}

Die 3-Monats-Prävalenz von Schmerzen allgemein nahm nach den vorliegenden Ergebnissen mit dem Alter der Kinder und Jugendlichen zu. Die höchsten Prävalenzen wurden im Alter von 14-17 Jahren ermittelt. Dieses Ergebnis steht in Übereinstimmung mit anderen Studien, in denen ebenfalls altersabhängige Anstiege der Prävalenz von Schmerzen ermittelt wurden $[1,2,3,14,15]$. $\mathrm{Zu}$ bedenken ist hier allerdings auch der Methodenwechsel von Fremd- (bei den 3- bis 10-jährigen) zu Selbstbeurteilung (bei den 11- bis 17jährigen). Möglicherweise lässt sich ein Teil des Anstiegs der Schmerzprävalenz durch die bessere Wahrnehmung eigener Schmerzen bei den 11- bis 17-Jährigen erklären.

Häufigste Lokalisationen waren in der Altersgruppe der 3- bis 10-Jährigen Bauchschmerzen, gefolgt von Kopf-, Bein- und Ohrenschmerzen. In der Gruppe der 11bis 17-Jährigen wurden Kopfschmerzen am häufigsten genannt, danach kamen Bauch-, Rücken-, Hals- und Beinschmerzen. In anderen Studien wurden ebenfalls in Übereinstimmung mit diesem Ergebnis altersabhängige Unterschiede in den Schmerzlokalisationen ermittelt [16, 17]. In Übereinstimmung mit den vorliegenden Studienergebnissen wurden insbesondere wiederkehrende Schmerzen auch in anderen Untersuchungen von älteren Kindern häufiger als von jüngeren Kindern berichtet $[1,3,14,18]$.

Schmerzen an mehr als einer Körperregion traten in der Gruppe der 3- bis 10Jährigen bei 44,6\% der Kinder und in der Gruppe der 11- bis 17-Jährigen bei 64,5\% auf. In Übereinstimmung mit diesem Ergebnis berichteten Petersen et al. [18] eine zunehmende Prävalenz von multiplen Schmerzen mit dem Alter der befragten Kinder. In einer niederländischen Studie berichtete etwa die Hälfte der befragten obis 18-jährigen Kinder und Jugendlichen Schmerzen mit mehr als einer Schmerzlokalisation [1]. In einer bundesdeutschen Studie an Schulkindern im Alter von 3-20 Jahren betrug die Prävalenz für
Schmerzen mit mehr als einer Lokalisation $66,3 \%$ [2].

Auch die Auftretenshäufigkeit von Schmerzen nahm nach den Ergebnissen dieses Studienteils mit dem Alter der Kinder und Jugendlichen zu. Diese Ergebnisse stehen in Übereinstimmung mit den Ergebnissen von Perquin et al. [1], die ebenfalls einen altersabhängigen Anstieg der Auftretenshäufigkeit von Schmerzen berichteten.

\section{Geschlechtsunterschiede}

Eine höhere Schmerzprävalenz bei Mädchen im Vergleich zu gleichaltrigen Jungen wurde in Übereinstimmung mit den vorliegenden Ergebnissen in vielen Studien berichtet $[1,2,3,13,15,19,20,21,22]$.

11- bis 17-jährige Mädchen berichteten signifikant häufiger als gleichaltrige Jungen wiederholte Schmerzen in den letzten 3 Monaten in den Lokalisationen Kopf, Rücken und Bauch gehabt zu haben. Dieses Ergebnis steht in Übereinstimmung mit den Ergebnissen anderer Studien, nach denen die Schmerzlokalisationen in Abhängigkeit von Alter und Geschlecht variieren [21, 23]. Auch die Auftretenshäufigkeit von Schmerzen war bei 11- bis 17-jährigen Mädchen höher als bei gleichaltrigen Jungen. Mädchen berichteten in einigen Untersuchungen ebenfalls häufiger eine höhere Frequenz von Schmerzen als gleichaltrige Jungen $[1,17]$.

Beziehungen von Schmerzen und pubertaler Entwicklung bei Jugendlichen wurden in einer Studie von LeResche et al. [24] untersucht. Dabei war sowohl für Mädchen als auch für Jungen die Pubertätsentwicklung ein besserer Prädiktor für Schmerzen als das Alter. Die Ursachen für die beobachteten Geschlechtsunterschiede in der Prävalenz und Auftretenshäufigkeit von Schmerzen sind gegenwärtig noch unklar. Hormonelle geschlechtsspezifische Unterschiede, insbesondere im Verlauf der Pubertätsentwicklung, eine höhere Symptomwahrnehmung auf Seiten der Mädchen sowie Auswirkungen sozialer Rollenerwartungen wurden unter anderem diskutiert $[18,25,26]$. Soweit es mit den vorliegenden Daten möglich ist, soll dieser Frage in späteren Analysen vertiefend nachgegangen werden. 
Inanspruchnahme medizinischer Leistungen

Mit jüngeren Kindern wird wegen Schmerzen häufiger zum Arzt gegangen, die Arztinanspruchnahme nimmt mit zunehmendem Alter ab. In Übereinstimmung mit den Ergebnissen anderer Studien nahm die Medikamenteneinnahme wegen Schmerzen jedoch mit dem Alter $\mathrm{zu}[27,28]$. Geschlechtsspezifische Unterschiede im Medikamentengebrauch werden insbesondere in oder nach der $\mathrm{Pu}$ bertät beobachtet [27]. Nach den Ergebnissen anderer Studien ist der Gebrauch von Schmerzmedikamenten bei Mädchen höher als bei Jungen $[27,28]$.

\section{Schlussfolgerungen und Ausblick}

Die Ergebnisse dieser Befragung bestätigen auf für Deutschland repräsentativer Ebene, dass Schmerzen bei Kindern und Jugendlichen ein ernst zu nehmendes Problem darstellen. Allerdings zeigen diese ersten Ergebnisse auch, dass sich hinter dem Phänomen Schmerzen bei Kindern und Jugendlichen Beschwerden unterschiedlicher Natur und Bedeutung verbergen. Neben einmaligen Schmerzen, die weder einen Arztbesuch noch Medikamenteneinnahme nach sich ziehen, werden von einem überraschend hohen Anteil von Kindern Schmerzen berichtet, die häufig auftreten und zu wiederholten Arztbesuchen bzw. häufiger Medikamenteneinnahme führen. Hält man sich die weite Verbreitung von chronischen Schmerzen bei Erwachsenen vor Augen, stellt sich die Frage, wann solche Chronifizierungsprozesse beginnen und welche Schlussfolgerungen sich daraus für die Präventionsarbeit bei Kindern ergeben.

Ziel weiterer Untersuchungen wird es sein, die durch Schmerzen im Allgemeinen und an speziellen Lokalisationen verursachten Beeinträchtigungen der Lebensqualität und der subjektiven Befindlichkeit zu quantifizieren und Schmerz auslösende Faktoren zu benennen. Es soll geprüft werden, ob Verhaltensweisen oder bestimmte äußere Umstände existieren, die mit vermehrtem oder vermindertem Auftreten von Schmerzen assoziiert sind, um damit gezielte Hinweise für die Prä- vention von Schmerzen im Kindes- und Jugendalter geben zu können.

\section{Korrespondierende Autorin}

\section{Dr. Ute Ellert}

Robert Koch-Institut

Abteilung für Epidemiologie und

Gesundheitsberichterstattung

Postfach 650261

13302 Berlin, BRD

E-Mail: u.ellert@rki.de

\section{Literatur}

1. Perquin $\mathrm{CW}$, Hazebroek-Kampschreur AA, Hunfeld $J A$ et al. (2000) Pain in children and adolescents: a common experience. Pain 87:51-58

2. Roth-lsigkeit A, Raspe HH, Stöven H et al. (2003) Schmerzen bei Kindern und Jugendlichen - Ergebnisse einer explorativen epidemiologischen Studie. Schmerz 17:171-178

3. Roth-Isigkeit A, Thyen U, Raspe HH et al. (2004) Reports of pain among German children and adolescents: an epidemiological study. Acta Paediatrica 93:258-263

4. Roth-Isigkeit A, Thyen U, Stöven H et al. (2005) Pain among children and adolescents: restrictions in daily living and triggering factors. Pediatrics 115 : 152-162

5. Bellach B-M, Ellert U, Radoschewski M (2000) Epidemiologie des Schmerzes - Ergebnisse des Bundes-Gesundheitssurveys 1998. Bundesgesundheitsbl Gesundheitsforsch Gesundheitsschutz 43:424-431

6. Frankenberg S, Pothmann R (1995) Epidemiologie von Kopfschmerzen bei Schulkindern. Psychomed 7:157-163

7. Kristjansdottir G (1996) Sociodemographic differences in the prevalence of self-reported stomach pain in school children. Eur J Pediatr 155:981-983

8. Chan S, Ryan MD (1992) Low-back pain in school children in the fifth and sixth grade. J Orthop Rheumatol 5:43-47

9. Perquin CW, Hazebroek-Kampschreur AA, Hunfeld JA et al. (2000) Chronic pain among children and adolescents: physician consultation and medication use. Clin J Pain 16:229-235

10. Kurth B-M (2007) Der Kinder- und Jugendgesundheitssurvey (KiGGS): Ein Überblick über Planung, Durchführung und Ergebnisse unter Berücksichtigung von Aspekten eines Qualitätsmanagements. Bundesgesundheitsbl Gesundheitsforsch Gesundheitsschutz 50:533-546

11. Kamtsiuris $P$, Lange $M$, Schaffrath Rosario $A$ (2007) Der Kinder- und Jugendgesundheitssurvey (KiGGS): Stichprobendesign, Response und Nonresponse-Analyse. Bundesgesundheitsbl Gesundheitsforsch Gesundheitsschutz 50:547-556

12. Hölling H, Kamtsiuris $P$, Lange $M$ et al. (2007) Der Kinder- und Jugendgesundheitssurvey (KiGGS): Studienmanagement und Durchführung der Feldarbeit. Bundesgesundheitsbl Gesundheitsforsch Gesundheitsschutz 50:557-566
13. Lange $M$, Kamtsiuris $P$, Lange $C$ et al. (2007) Messung soziodemographischer Merkmale im Kinderund Jugendgesundheitssurvey (KiGGS) und ihre Bedeutung am Beispiel der Einschätzung des allgemeinen Gesundheitszustands. Bundesgesundheitsbl Gesundheitsforsch Gesundheitsschutz 50:578-589

14. Kristjansdottir $G$ (1997) Prevalence of pain combinations and overall pain: a study of headache, stomach pain and back pain among schoolchildren. Scand J Soc Med 25:58-63

15. Petersen S, Bergstrom E, Brulin C (2003) High prevalence of tiredness and pain in young schoolchildren. Scand J Public Health 31:367-374

16. Fichtel A, Larsson B (2002) Psychosocial impact of headache and comorbidity with other pains among Swedish school adolescents. Headache 42:766-775

17. Larsson B, Sund AM (2007) Emotional/behavioural, social correlates and one-year predictors of frequent pains among early adolescents: Influences of pain characteristics. Eur J Pain 11:57-65

18. Petersen S, Brulin C, Bergstrom E (2006) Recurrent pain symptoms in young schoolchildren are often multiple. Pain 121:145-150

19. Watson KD, Papageorgiou AC, Jones GT et al. (2002) Low back pain in schoolchildren: occurrence and characteristics. Pain 97:87-92

20. Leonardsson-Hellgren M, Gustavsson UM, Lindblad U (2001) Headache and associations with lifestyle among pupils in senior level elementary school. Scand J Prim Care 19:107-111

21. Hirsch C, John MT, Schaller HG, Turp JC (2006) Painrelated impairment and health care utilization in children and adolescents: a comparison of orofacial pain with abdominal pain, back pain, and headache. Quintessence Int 37:381-390

22. Rhee H, Miles MS, Halpern CT, Holditch-Davis D (2005) Prevalence of recurrent physical symptoms in U.S. adolescents. Pediatr Nurs 31:314-319

23. Torsheim T, Ravens-Sieberer U, Hetland J et al. (2006) Cross-national variation of gender differences in adolescent subjective health in Europe and North America. Soc Sci Med 62:815-827

24. LeResche L, Mancl LA, Drangsholt MT et al. (2005) Relationship of pain and symptoms to pubertal development in adolescents. Pain 118:201-209

25. Pennebaker JW, Watson D (1991) The physiology of somatic symptoms. In: Kirmayer LJ, Robbins JM (eds) Current concepts of somatization: research and clinical perspectives. American Psychiatric Press, Washington, DC, pp 21-35

26. McGrath PA (1994) Psychological aspects of pain perception. Arch Oral Biol 39(Suppl):55S-62S

27. Roe CM, McNamara AM, Motheral BR (2002) Gender- and age-related prescription drug use patterns. Ann Pharmacother 36:30-39

28. Holstein BE, Holme Hansen E, Due P, Birna Almarsdottir A (2003) Self-reported medicine use among 11- to 15-year-old girls and boys in Denmark 19881998. Scand J Public Health 31:334-341 